\title{
Muoniated spin probes in the discotic liquid crystal HHTT: Rapid electron spin relaxation in the hexagonal columnar and isotropic phases
}

\author{
Iain McKenzie,,${ }^{1,2, *}$ Andrew N. Cammidge,${ }^{3}$ Hemant Gopee,${ }^{3}$ Herbert Dilger ${ }^{4}$ Robert Scheuermann, ${ }^{5}$ \\ Alexey Stoykov, ${ }^{5}$ and Upali A. Jayasooriya ${ }^{3}$ \\ ${ }^{1}$ Centre for Molecular and Materials Science, TRIUMF, 4004 Wesbrook Mall, Vancouver, British Columbia, Canada, V6T $2 A 3$ \\ ${ }^{2}$ Department of Chemistry, Simon Fraser University, 8888 University Drive, Burnaby, British Columbia, Canada, V5A 1S6 \\ ${ }^{3}$ School of Chemistry, University of East Anglia, NR4 7TJ, Earlham Road, Norwich, United Kingdom \\ ${ }^{4}$ Institut für Physikalische Chemie, Universität Stuttgart, Pfaffenwaldring 55, D-70569 Stuttgart, Germany \\ ${ }^{5}$ Laboratory for Muon Spectroscopy, Paul Scherrer Institute, CH-5232, Villigen, Switzerland
}

(Received 5 September 2012; published 22 January 2013)

\begin{abstract}
Avoided level crossing muon spin resonance (ALC- $\mu \mathrm{SR}$ ) spectroscopy was used to study radicals produced by the addition of the light hydrogen isotope muonium $(\mathrm{Mu})$ to the discotic liquid crystal (DLC) 2,3,6,7,10,11hexahexylthiotriphenylene (HHTT). Mu adds to the secondary carbon atoms of HHTT to produce a substituted cyclohexadienyl radical, whose identity was confirmed by comparing the measured hyperfine coupling constants with values obtained from DFT calculations. ALC- $\mu$ SR spectra were obtained in the isotropic (I), hexagonal columnar $\left(\mathrm{Col}_{h}\right)$, helical $(\mathrm{H})$, and crystalline $(\mathrm{Cr})$ phases. In the $\mathrm{I}$ and $\mathrm{Col}_{\mathrm{h}}$ phases the radicals, which are incorporated within the stacks of HHTT molecules as isolated paramagnetic defects, undergo extremely rapid electron spin relaxation, on the order of a hundredfold faster than in the $\mathrm{H}$ or $\mathrm{Cr}$ phases. The electron spin relaxation rate increases significantly with increasing temperature and appears to be caused by the liquidlike motion within the columns, which modulates the overlap between the $\pi$ system of the radical and the $\pi$ systems of the neighboring HHTT molecules, and hence, the hyperfine coupling constants. Rapid electron spin relaxation should occur for any $\pi$ radical incorporated within the columns of a DLC, which may limit the utility of DLCs for future spin-based technologies.
\end{abstract}

DOI: 10.1103/PhysRevE.87.012504

PACS number(s): 64.70.mj, 76.30.Rn, 76.75.+i

\section{INTRODUCTION}

Liquid crystals are materials with phases that have anisotropic properties normally found in solids and a degree of fluidity characteristic of liquids [1-3]. The molecules of a liquid crystal show, on average, a significant preferred orientational order due to anisotropy in the shape of the molecules and the resulting intermolecular forces. Thermotropic liquid crystals are commonly classified in terms of the shape of the molecules, with calamitic liquid crystals made up of long and narrow molecules and discotic liquid crystals (DLCs) made up of disk-shaped molecules [4]. DLCs generally consist of a rigid planar conjugated core and flexible hydrocarbon side chains and can form a wide variety of mesophases, including nematic, lamellar, and columnar phases. The columnar phases are classified according to the symmetry of the columns and the nature of the intracolumn order. Examples are the hexagonal columnar mesophase $\mathrm{Col}_{h}$, the columnar rectangular mesophase $\mathrm{Col}_{\mathrm{r}}$, the columnar oblique mesophase $\mathrm{Col}_{\mathrm{ob}}$, and the helical phase $\mathrm{H}$.

One of the most widely studied DLCs is $2,3,6,7,10,11$ hexakis(hexylthio)triphenylene (HHTT), which has a triphenylene core and six thioether substituents. The structure and phase transition temperatures of HHTT are shown in Fig. 1. The phase transition temperatures depend on whether the sample is being heated or cooled [5]. The crystalline (Cr) phase of HHTT has a monoclinic unit cell containing four molecules separated by $3.937 \AA$ [6]. Adjacent molecules are

*iain.mckenzie@triumf.ca twisted $180^{\circ}$ with respect to each other and laterally displaced by approximately $0.5 \AA$. HHTT forms two mesophases whose properties have been determined by high-resolution $\mathrm{x}$-ray diffraction [6,7]. In the hexagonal $(\mathrm{H})$ phase the HHTT molecules are ordered along the columns, with one out of three columns displaced along the columnar axis by half the intermolecular distance, hence forming a $\sqrt{3} \times \sqrt{3} \mathrm{R}$ $30^{\circ}$ (or honeycomb) superlattice in the hexagonal plane. The intracolumn spacing is $3.638 \AA$ and adjacent molecules are twisted by $45.5^{\circ}$ with respect to each other. HHTT is the only material in which a $\mathrm{H}-\mathrm{Col}_{h}$ transition has been observed. The $\mathrm{Col}_{\mathrm{h}}$ phase is made up of a two-dimensional triangular array of columns with fluidlike intracolumn ordering and no longrange intercolumn correlation. HHTT becomes a very viscous isotropic liquid (I) above 363-365 K. The columnar arrangement of the aromatic cores in these mesophases and the typical intracolumn distances of about $3.5 \AA$ can result in overlap of the $\pi^{*}-\pi^{*}$ lowest-unoccupied molecular orbitals (LUMOs), which gives a low-dimensional conduction pathway and high charge mobility. The unique structural and electronic properties of discotic liquid crystals have applications in molecular electronics and high-efficiency organic voltaics. HHTT is notable due to the high charge mobility $\left(0.4 \mathrm{~cm}^{2} \mathrm{~V}^{-1} \mathrm{~s}^{-1}\right.$ in the $\mathrm{Cr}$ phase and $0.1 \mathrm{~cm}^{2} \mathrm{~V}^{-1} \mathrm{~s}^{-1}$ in the $\mathrm{H}$ phase), which is higher than in almost any other organic material $[8,9]$.

Most of the studies of HHTT have used techniques that examine the bulk properties of the material but it is a longstanding goal to better understand the behavior of the molecules within the DLC. Dvinskikh et al. studied the dynamics of the HHTT molecules using ${ }^{1} \mathrm{H}$ and ${ }^{13} \mathrm{C}$ nuclear magnetic resonance (NMR) [5,10]. The linewidths of the 


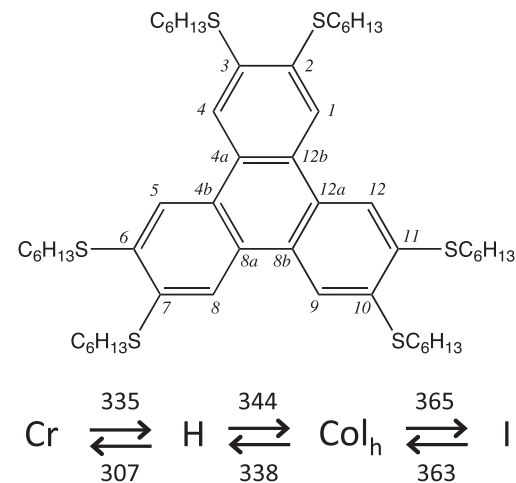

FIG. 1. The structure of HHTT with the IUPAC numbering of the carbon atoms of the triphenylene ring and its transition temperatures (K) for heating and cooling rates of $5 \mathrm{~K} \mathrm{~min}^{-1}$ [5].

features in both the ${ }^{1} \mathrm{H}$ and ${ }^{13} \mathrm{C}$ NMR spectra decreased with increasing temperature, with a narrow spectrum in the isotropic phase. The ${ }^{1} \mathrm{H}-{ }^{13} \mathrm{C}$ dipolar coupling in the $\mathrm{Cr}$ phase indicated that the triphenylene core and the inner methylene groups are immobile, while the methyl and outer methylene groups exhibit partial mobility. The $\mathrm{H}$ phase is also a solid phase, with the difference between the $\mathrm{Cr}$ and $\mathrm{H}$ phases being an increased mobility of the aliphatic chains in the $\mathrm{H}$ phase. The aromatic core of HHTT undergoes rotation around the molecular symmetry axis in the $\mathrm{Col}_{\mathrm{h}}$ phase on a time scale of less than $\sim 10^{-4} \mathrm{~s}$ and the director is oriented perpendicular to the magnetic field. HHTT is highly ordered in the $\mathrm{Col}_{\mathrm{h}}$ phase with the order parameter $(S)$ equal to 0.80 at $345 \mathrm{~K}$ and 0.65 at $363 \mathrm{~K}$ [5].

Additional information about the behavior of HHTT at the molecular level can be obtained by spin labeling the HHTT molecules with positive muons and studying the resulting radicals with muon spectroscopic techniques collectively known as $\mu \mathrm{SR}$ for muon spin rotation, resonance, and relaxation [11]. This is similar to the introduction of stable nitroxide spin probes into soft matter systems, except that the muoniated radicals are formed in situ and have similar structures to the parent molecules [12]. The positive muon $\left(\mu^{+}\right)$is an elementary particle with a spin of $1 / 2$, a lifetime of $2.2 \mu \mathrm{s}$, a magnetic moment 3.183 times that of the proton and a mass approximately one-ninth that of the proton. Beams of mono-energetic muons with spin polarization close to $100 \%$ are available at several facilities (Paul Scherrer Institut, Switzerland; TRIUMF, Canada; ISIS, United Kingdom; J-PARC, Japan). The muon decays into a positron and two neutrinos and the positron is emitted preferentially along the direction of the muon's spin, providing a convenient means of monitoring the temporal evolution of the muon's spin. Muons can pick up an electron during the radiolysis process to form a one-electron atom called muonium $\left(\mathrm{Mu}=\left[\mu^{+}, \mathrm{e}^{-}\right]\right)$, which is considered to be a light isotope of hydrogen as its reduced mass and ionization energy are within $0.5 \%$ those of $\mathrm{H}$. The addition of $\mathrm{Mu}$ to an unsaturated bond produces a radical (called a muoniated radical) where the muon takes the position of a proton attached to an atom next to the radical center. There is a hyperfine interaction between the unpaired electron and nuclei with spin greater than zero. The strength of this interaction, the hyperfine coupling constant (hfcc), $A_{\mathrm{X}}$, is proportional to the unpaired spin density at the nucleus $|\psi(0)|^{2}$ and is the sum of the isotropic or Fermi contact term, $A_{\mathrm{X}}^{\text {iso }}$, and the anisotropic or dipolar term (given the symbol $B_{\mathrm{X}}$ for rhombic symmetry and $D_{\mathrm{X}}$ for axial symmetry). The structure of muoniated radicals can be determined by measuring the muon and as many nuclear hfccs as possible, as this maps out the singly occupied molecular orbital (SOMO), and comparing these values with hfccs obtained from $a b$ initio calculations of the possible structures. The magnitude and temperature dependence of the hfccs can provide information about the configuration and conformation of free radicals as well as the local environment.

The muon and nuclear hfccs can be measured using several spectroscopic techniques based on the anisotropic radioactive decay of the muon, including avoided level crossing muon spin resonance (ALC- $\mu \mathrm{SR}$ ). In the ALC- $\mu$ SR experiment the sample is located in a magnetic field parallel to the polarization of the muon beam. In high magnetic fields the spin states of a radical are usually products of the pure Zeeman states of the $I>0$ nuclei and the unpaired electron. The total spin quantum number of a spin state, $M$, is the sum of the spin quantum numbers of the unpaired electron and the nuclei in the radical. There is no evolution of the muon spin with time and the asymmetry is independent of the applied field. This is not the case near avoided crossings of magnetic energy levels with different muon and nuclear spin orientations. At specific values of the applied field, nearly degenerate pairs of spin states can be mixed through the isotropic and anisotropic components of the hyperfine interaction. The spin states in this situation are mixtures of Zeeman states and the muon polarization oscillates between the two mixing states, leading to a loss of muon spin polarization and a resonantlike change in the asymmetry as the magnetic field is swept. There are three types of ALC resonances observed in electron-muon-nuclear systems, which are characterized by the selection rule $|\Delta M|=0,1$, and 2 , and are referred to as $\Delta_{0}, \Delta_{1}$, and $\Delta_{2}$ resonances, respectively. The $\Delta_{0}$ resonance arises from mixing between levels with opposite muon and nuclear spins due to the isotropic portion of the spin Hamiltonian. These resonances are observed for muoniated radicals in solids, liquids or gases and are used to determine nuclear hfccs, provided the muon hfcc is known. The resonance field for a $\Delta_{0}$ resonance $\left(B_{\mathrm{res}}^{\Delta_{0}}\right)$ is given by

$$
B_{\mathrm{res}}^{\Delta_{0}}=\frac{1}{2}\left(\frac{A_{\mu}-A_{\mathrm{X}}}{\gamma_{\mu}-\gamma_{\mathrm{X}}}-\frac{A_{\mu}+A_{\mathrm{X}}}{\gamma_{\mathrm{e}}}\right),
$$

where $A_{\mu}$ and $A_{\mathrm{X}}$ are the muon and nuclear hfccs, respectively, and $\gamma_{\mu}$ and $\gamma_{\mathrm{X}}$ are the muon and nuclear gyromagnetic ratios, respectively. The nuclear hfccs can be determined by measuring $B_{\text {res }}^{\Delta_{0}}$, if the muon hfcc is known.

The $\Delta_{1}$ resonance arises from mixing between spin states with the same electron and nuclear spins but different muon spin. These spin states are only mixed in the presence of anisotropy, so the presence of a $\Delta_{1}$ resonance can be considered to be diagnostic of a frozen state or of anisotropic motion. The resonances are averaged out by isotropic motion on a critical time scale given by the inverse of the hyperfine anisotropy (typically $50 \mathrm{~ns}$ for muoniated cyclohexadienyl 
radicals). The $\Delta_{1}$ resonance field ( $\left.B_{\text {res }}^{\Delta_{1}}\right)$ is given by

$$
B_{\mathrm{res}}^{\Delta_{1}}=\frac{1}{2}\left(\frac{A_{\mu}}{\gamma_{\mu}}-\frac{A_{\mu}}{\gamma_{\mathrm{e}}}\right),
$$

so the $\Delta_{1}$ resonance can be used to measure the muon hfcc of radicals in anisotropic environments. The $\Delta_{1}$ resonance is very sensitive to the motion of the radical but is approximately Lorentzian when the hyperfine anisotropy is small compared with the spin relaxation rate. Kreitzman and Roduner have investigated the effect of rotational diffusion, chemical reaction, and electron spin relaxation on the shape of the $\Delta_{1}$ and $\Delta_{0}$ resonances [13].

The $\Delta_{2}$ resonance is a double quantum transition between spin states with opposite muon and nuclear spins and is specific to anisotropic environments. It is very weak, narrow, and has been rarely observed.

Muon spin spectroscopy has been used to study a wide range of soft matter systems, such as the calamitic liquid crystals 4-n'-pentyl-4-cyanobiphenyl (5CB) [14-16], 4-n'-octyl-4-cyanobiphenyl (8CB) [17], cholesterol nonanoate [18], 4-(trans-4-pentylcyclohexyl)benzonitrile (PCH5) [19], and the partitioning of cosurfactants in lamellar phase dispersions [20-23]. ALC- $\mu$ SR has been used to study the DLC 2,3,6,7,10,11-hexahexyloxytriphenylene (HAT6) [24]. Muoniated radicals were formed by $\mathrm{Mu}$ addition to the unsaturated secondary carbon of the triphenylene ring of HAT6. In the $\mathrm{Col}_{\mathrm{h}}$ and I phases these radicals appear to exist in two distinct environments. The majority of the radicals reside within the columns, where there is rapid electron spin relaxation that results in a very broad and intense $\Delta_{1}$ resonance. Some of the muoniated radicals are slipped between the columns and are highly mobile, which results in narrow $\Delta_{1}$ and methylene proton $\Delta_{0}$ resonances.

$\mathrm{Mu}$ adds exclusively to the unsaturated secondary carbons of HHTT's triphenylene core (carbons 1,4,5,8,9,12) to give the radical shown in Fig. 2, which will be referred to as $\mathrm{Mu}$-1-HHTT. Several studies of the Mu adducts of polyaromatic hydrocarbons have shown that $\mathrm{Mu}$ does not add to tertiary carbons because this causes significant distortion of the ring system and is energetically unfavorable [25-27]. In these experiments the HHTT molecules were partially aligned by cooling from the isotropic phase in a large $(2.5 \mathrm{~T})$ magnetic field. The motivation for this study is to probe the dynamics of the HHTT at the molecular level and to determine how an isolated paramagnetic defect interacts with neighboring molecules within the HHTT columns.

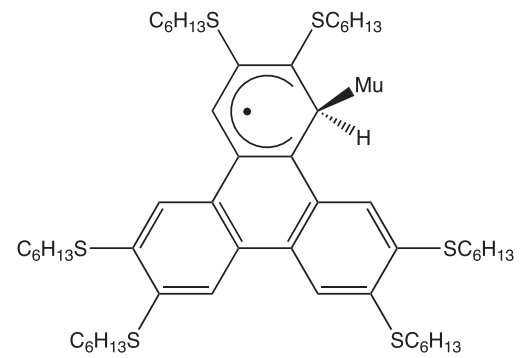

FIG. 2. The structure of the Mu adduct of HHTT (Mu-1-HHTT).

\section{EXPERIMENT}

HHTT was prepared following the procedure outlined by Kohne et al. [28]. Oxygen, which can broaden the resonances in the ALC- $\mu$ SR spectra by Heisenberg spin exchange [29], was removed by melting the sample in nitrogen atmosphere and bubbling $\mathrm{N}_{2}$ through the liquid for one hour, after which the sample was sealed in brass cells with an internal volume of $17 \mathrm{~mL}$ and $50 \mu \mathrm{m}$ titanium foil windows. The ALC- $\mu$ SR experiments were performed using the ALC spectrometer at the $\pi \mathrm{E} 3$ beam line of the Paul Scherrer Institute in Villigen, Switzerland. ALC- $\mu$ SR were obtained between 0.2 and $2.5 \mathrm{~T}$ with a step size of $5 \mathrm{mT}$ using a superconducting solenoid magnet.

The sample was allowed to equilibrate for 0.5 hours before measurements were made and each magnetic field scan took approximately 2.5 hours, with a minimum of two scans obtained per temperature, which were combined to produce the ALC- $\mu$ SR spectrum. The ALC- $\mu$ SR spectra were recorded going from $377 \mathrm{~K}$ to $302 \mathrm{~K}$ at $5 \mathrm{~K}$ intervals then down to $272 \mathrm{~K}$ at $10 \mathrm{~K}$ intervals. The phase transition temperatures depend on the thermal history of the sample and based on the changes in the ALC- $\mu$ SR spectra (discussed below) we propose that they occurred at $\sim 363 \mathrm{~K}\left(\mathrm{I}-\mathrm{Col}_{\mathrm{h}}\right), \sim 335 \mathrm{~K}\left(\mathrm{Col}_{\mathrm{h}}-\mathrm{H}\right)$, and $\sim 305 \mathrm{~K}$ (H-Cr). The transition temperatures established by ALC- $\mu \mathrm{SR}$ experiments on slow cooling (ca $0.2 \mathrm{~K} \mathrm{~min}^{-1}$ ) are in broad agreement with values obtained using differential scanning calorimetry at a cooling rate of $0.5 \mathrm{~K} \mathrm{~min}^{-1}(361 \mathrm{~K}, 331 \mathrm{~K}$, and $303 \mathrm{~K}$, respectively).

The ALC- $\mu$ SR spectra have a large field-dependent background that is very sensitive to the stopping position of the muons. The spectra in the $\mathrm{Cr}$ and $\mathrm{H}$ phases were fit using Lorentzian functions for the resonances and a fifth-order polynomial for the background. The background determined at $332 \mathrm{~K}$ (H phase) was subtracted from the spectra in the $\mathrm{Col}_{\mathrm{h}}$ and I phases. The ALC- $\mu$ SR spectra in the $\mathrm{Col}_{\mathrm{h}}$ and I phases were fit with a single Lorentzian and no background terms from 1.0 to $2.5 \mathrm{~T}$. The least-squares fitting was performed using the MINUIT function minimization library in the ROOT package from CERN [30].

$A b$ initio calculations were performed using the Gaussian 03 package of programs [31]. The structures were optimized with the unrestricted B3LYP density functional and the 6-311G(d,p) basis set. The $\mathrm{C}_{6} \mathrm{H}_{13}$ groups of HHTT were replaced with methyl groups to reduce the computational cost and these were constrained to lie in the plane of the triphenylene ring. The hfccs of an isolated Mu-1-HHTT radical were calculated using the unrestricted PBE0 density functional with the EPR-II basis set for $\mathrm{C}$ and $\mathrm{H}$ atoms and the 6-311G(d,p) basis set for $\mathrm{S}$ atoms. The reduced muon hfcc was obtained by multiplying the calculated hfcc by a factor of 1.20 to account for the "intrinsic isotope effect" that results from the anharmonicity of the $\mathrm{C}-\mathrm{H}$ stretching vibration, and the higher zero-point energy of the muoniated isotopomer. The "secondary isotope effect" was included by multiplying the methylene proton hfcc by a factor of 0.94. This computational methodology was demonstrated to be suitable for the $\mathrm{Mu}$ adducts of polyaromatic hydrocarbons $[26,27]$. Calculations were also performed for a Mu-1-HHTT radical with two neighboring HHTT molecules separated by $3.5 \AA$ using the molecular structures calculated for isolated 
molecules. The hfccs for this system were calculated with the unrestricted B3LYP density functional and the smaller 6-31G basis set.

\section{RESULTS AND DISCUSSION}

\section{A. DFT calculations}

The optimized structure of Mu-1-HHTT is shown in Fig. 3 with the calculated muon and proton hfecs (isotropic and dipolar) and the corresponding isotropic resonance fields. The majority of the unpaired electron spin density is located on the six-membered ring to which $\mathrm{Mu}$ has added. The calculated muon and methylene proton hfccs are slightly lower than the corresponding hfccs in the structurally similar $\mathrm{Mu}$ adducts of triphenylene $\left(A_{\mu}=422.10 \mathrm{MHz}\right)$ or HAT6 $\left(A_{\mu}=420.29\right.$ $\mathrm{MHz}$ ) calculated at the UB3LYP/6-311G(d,p) level [24].

Senthilkumar et al. have shown that the charge transfer integral for methylthio-substituted triphenylenes, which is proportional to the probability of electron tunneling between two neighboring molecules, varies considerably with the twist angle but also drops off exponentially with the intermolecular distance [33]. Calculations were performed as a function of the angle between the $\mathrm{Mu}-1-\mathrm{HHTT}$ radical and the neighboring HHTT molecules to determine the angular dependence of spin density transfer to the neighboring molecules. For $\mathrm{Mu}-$ 1-HHTT and HHTT we define a vector going from the center-of-mass to the midpoint of the $\mathrm{C} 2-\mathrm{C} 3$ bond. The angle between the two rings, $\theta$, is defined to be the angle between these two vectors. The spin density transferred from $\mathrm{Mu}-1-\mathrm{HHTT}$ to the neighboring HHTT molecules depends on the ring conformations, with the maximum amount of spin density transferred $(3.09 \%)$ when both rings are at an angle of $\theta=0^{\circ}$ and the minimum amount of spin density transferred $(1.65 \%)$ when both rings are at an angle of $\theta=120^{\circ}$. The minimum energy geometry, which occurs when both HHTT

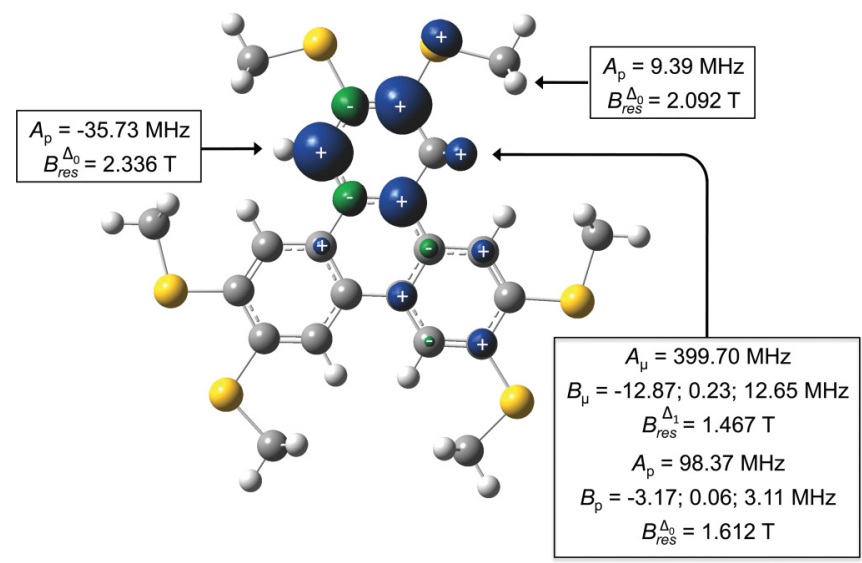

FIG. 3. (Color online) The optimized structure and distribution of unpaired electron spin density in the Mu-1-HHTT radical. Dark gray atom $=$ carbon, yellow (light gray) atom $=$ sulfur, white atom $=$ hydrogen/muonium. Positive spin density is blue and negative spin density is green (isovalue $=0.004$ ). Calculated $A_{\mu}$ and $A_{\mathrm{p}}$ values greater than $|5| \mathrm{MHz}$ are reported along with the $\Delta_{1}$ and $\Delta_{0}$ resonance fields. The dipolar hyperfine coupling constants $\left(B_{\mu}\right.$ and $B_{\mathrm{p}}$ ) are reported for the muon and methylene proton. The computational method is described in the text. molecules are at $\theta=120^{\circ}$, is $3.88 \mathrm{eV}$ lower in energy than the structure with both rings at an angle of $\theta=0^{\circ}$.

\section{B. Analysis of the ALC- $\mu$ SR spectra}

The ALC- $\mu$ SR spectra of DLC are unlike those of the multitude of organic radicals that have been previously studied. The raw and background-subtracted ALC- $\mu$ SR spectra in the $\mathrm{Cr}, \mathrm{H}, \mathrm{Col}_{\mathrm{h}}$, and I phases are shown in Fig. 4. There is a very broad approximately Lorentzian-shaped resonance around $1.30 \mathrm{~T}$ in the $\mathrm{Col}_{\mathrm{h}}$ and I phases that is assumed to be the $\Delta_{1}$ resonance for the $\mathrm{Mu}-1-\mathrm{HHTT}$ radical based on the similarity between the experimental and DFT calculated resonance field. The low field side of the resonance is complicated by the repolarization, which is why only data between 1.0 and $2.5 \mathrm{~T}$ were analyzed. The presence of a $\Delta_{1}$ resonance in the $\mathrm{Col}_{\mathrm{h}}$ and I phases indicates that the rotational correlation time of Mu-1-HHTT is greater than $\sim 80 \mathrm{~ns}$, which is unsurprising since HHTT is very viscous.

$A_{\mu}$ was calculated from the resonance field using Eq. (2) and was found to increase linearly as the temperature was lowered, with a small discontinuity at the $\mathrm{Col}_{\mathrm{h}}-\mathrm{I}$ phase transition (Fig. 5). The $\Delta_{1}$ resonance is very broad and intense and its width decreases as the temperature was lowered, with a discontinuity and change in slope at the $\mathrm{Col}_{\mathrm{h}}$-I phase transition (Fig. 6). The amplitude of the $\Delta_{1}$ resonance goes through a maximum at the $\mathrm{Col}_{\mathrm{h}}$-I phase transition (Fig. 7). The methylene proton $\Delta_{0}$ resonance of Mu-1-HHTT was not observed.

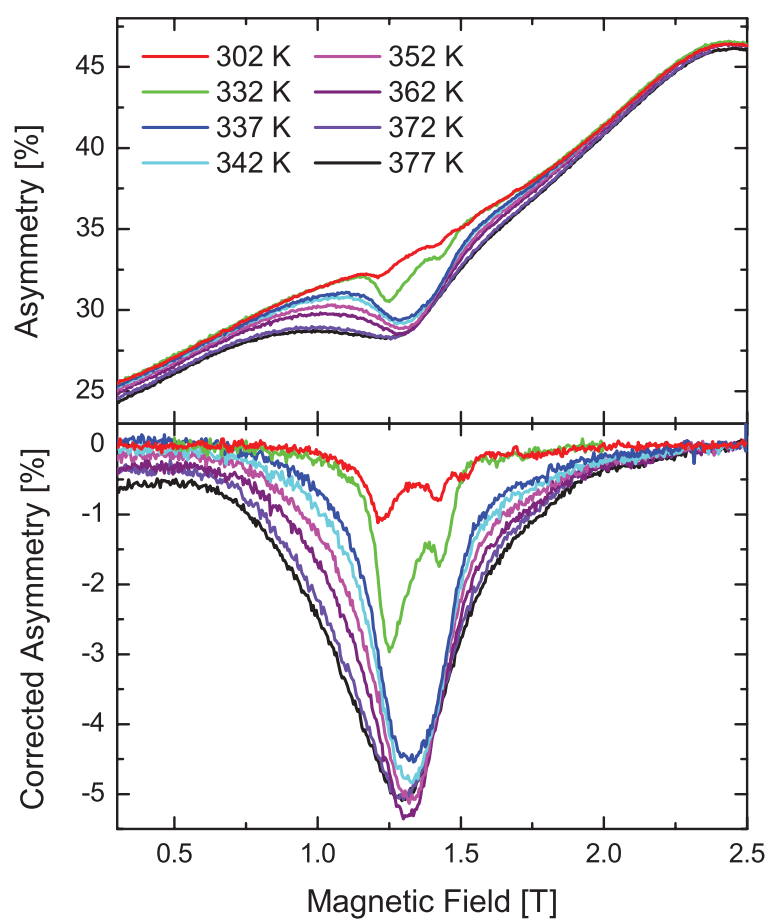

FIG. 4. (Color online) (top) Raw ALC- $\mu$ SR spectra of HHTT as a function of temperature in the $\mathrm{Cr}, \mathrm{H}, \mathrm{Col}_{\mathrm{h}}$, and I phases. (bottom) Background-subtracted ALC- $\mu$ SR spectra of HHTT as a function of temperature in the $\mathrm{Cr}, \mathrm{H}, \mathrm{Col}_{\mathrm{h}}$, and I phases. In both the top and bottom figures the spectrum at $302 \mathrm{~K}$ is the uppermost curve and the spectrum at $377 \mathrm{~K}$ is the lowermost curve. 


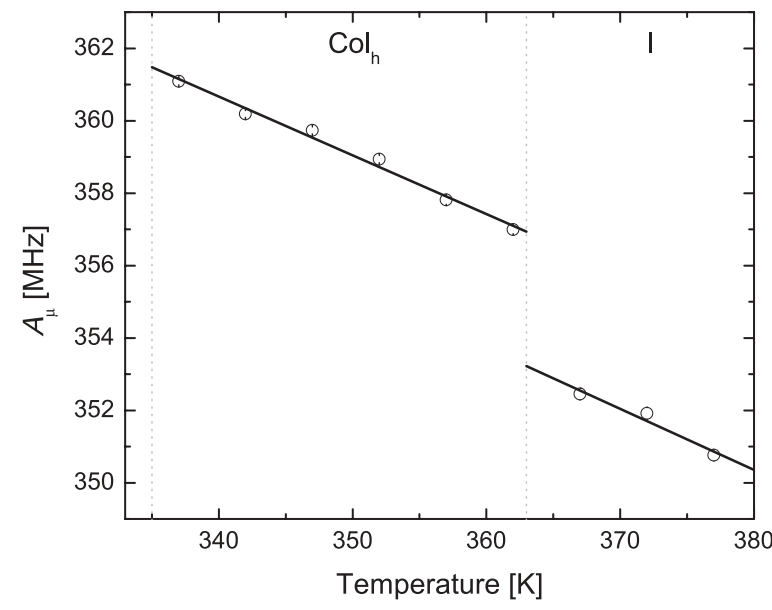

FIG. 5. Muon hyperfine coupling constant of the Mu adduct of HHTT as a function of temperature. The dotted vertical lines denote the $\mathrm{H}-\mathrm{Col}_{\mathrm{h}}$ and $\mathrm{Col}_{\mathrm{h}} \mathrm{I}$ phase transitions.

The discontinuity of $A_{\mu}$ at the $\mathrm{Col}_{\mathrm{h}}$-I phase transition is consistent with the partial alignment of the columns in the $\mathrm{Col}_{\mathrm{h}}$ phase perpendicular to the magnetic field. The closely related DLC HAT6 is aligned in a field of $\sim 1.42 \mathrm{~T}$ [32]. The rotation rate of HHTT is not sufficient to generate an axially symmetric hyperfine tensor, however we can treat an ensemble of aligned radicals as having an effective axially symmetric hyperfine tensor, with the unique component, $D_{\mu}^{\|}$, along the local director. The difference between the muon hfcc in the $\mathrm{Col}_{\mathrm{h}}$ and I phases depends on $D_{\mu}^{\|}$, the angle between the rotation axis and the applied magnetic field, $\theta$, and the fraction of molecules at a given angle, $f(\theta)$,

$$
A_{\mu}^{\mathrm{Col}_{\mathrm{h}}}-A_{\mu}^{\mathrm{I}}=\int_{0}^{\pi} \frac{1}{2} D_{\mu}^{\|}\left(3 \cos ^{2} \theta-1\right) f(\theta) 2 \pi \sin (\theta) d \theta .
$$

The order parameter of HHTT is 0.65 at $363 \mathrm{~K}$ (close to the clearing point), so the angular distribution function can be estimated using the Maier-Saupe model [1]. From this

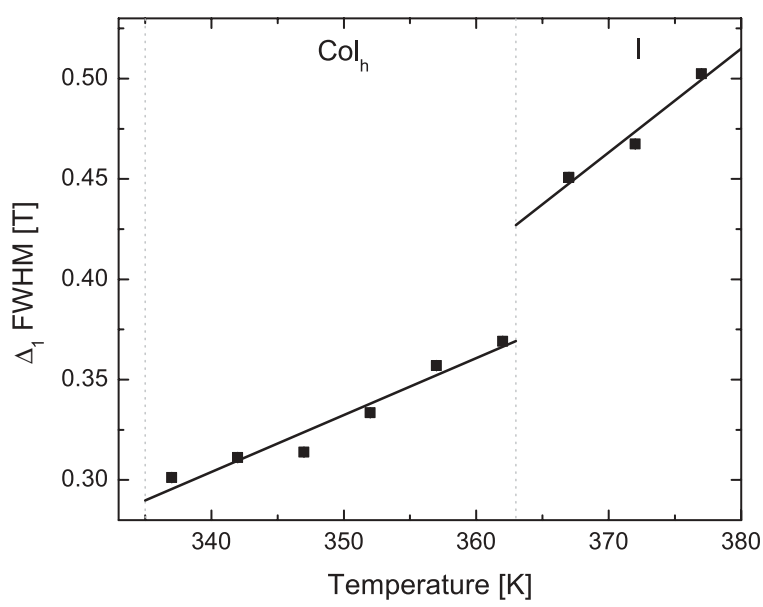

FIG. 6. Temperature dependence of the FWHM of the $\Delta_{1}$ resonance of the Mu-1-HHTT radical as a function of temperature in the $\mathrm{Col}_{h}$ and I phases. The dotted vertical lines denote the $\mathrm{H}-\mathrm{Col}_{h}$ and $\mathrm{Col}_{\mathrm{h}} \mathrm{-I}$ phase transitions.

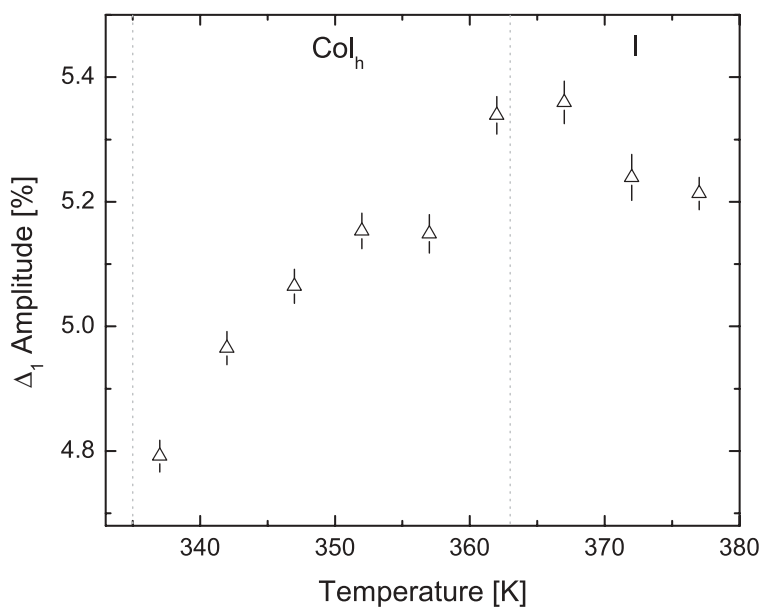

FIG. 7. Temperature dependence of the amplitude of the $\Delta_{1}$ resonance of the Mu-1-HHTT radical as a function of temperature in the $\mathrm{Col}_{h}$ and I phases. The dotted vertical lines denote the $\mathrm{H}-\mathrm{Col}_{h}$ and $\mathrm{Col}_{\mathrm{h}}$-I phase transitions.

we calculate that $A_{\mu}^{\mathrm{Col}_{h}}-A_{\mu}^{\mathrm{I}}$ should be $\sim-1.22 D_{\mu}^{\|}$, which means that $D_{\mu}^{\|} \sim-3.0(1) \mathrm{MHz}$. The $D_{\mu}^{\|}$obtained from the DFT calculations $-9.2 \mathrm{MHz}$, with the lower experimental dipolar hfcc being consistent with considerable wobbling of the molecules about their mean orientation and only partial alignment of the columns in the magnetic field.

The ALC- $\mu$ SR spectra of HHTT are unlike those of HAT6 in that there are no narrow resonances superimposed on a broad and intense $\Delta_{1}$ resonance. In HAT6 we assigned the narrow resonances to highly mobile radicals that were slipped in between the columns. The absence of narrow resonances in the ALC- $\mu$ SR spectra of HHTT indicates that all the radicals are incorporated into columns.

The ALC- $\mu$ SR spectra change dramatically between 332 and $337 \mathrm{~K}$, which we propose corresponds to the $\mathrm{H}-\mathrm{Col}_{\mathrm{h}}$ phase transition. The ALC- $\mu$ SR spectra in the $\mathrm{Cr}$ and $\mathrm{H}$ phases are reminiscent of the spectra obtained in other soft matter systems in that the resonance FWHM linewidths are between 50 and $120 \mathrm{mT}$. We observed multiple smaller resonances centered at $\sim 1.3 \mathrm{~T}$, which split and decreased in amplitude as the sample was cooled. There are three large resonances at 1.2284(9), 1.415(1), and 1.517(2) $\mathrm{T}$ in the ALC- $\mu$ SR spectrum of HHTT at $302 \mathrm{~K}$ (Cr phase) and we propose that these are overlapping $\Delta_{1}$ and methylene proton $\Delta_{0}$ resonances of Mu-1-HHTT radicals in different environments. The resonances at $\sim 1.42$ and $1.52 \mathrm{~T}$ are the $\Delta_{1}$ and $\Delta_{0}$ resonances, respectively, for one environment, giving $A_{\mu} \gamma_{p} / A_{p} \gamma_{\mu}=1.190(7)$, which is a reasonable value for muoniated cyclohexadienyl-type radicals. The resonance at $\sim 1.23 \mathrm{~T}$ is the $\Delta_{1}$ resonance for another environment and we propose that there is an additional $\Delta_{0}$ resonance at $1.33 \mathrm{~T}$ that is hidden under the other resonances. Splitting of the resonances could be due to differences in the spin density transferred to neighboring molecules, different orientations of the radicals with respect to the magnetic field or a combination of both. These possibilities have been investigated with the DFT calculations. The splitting of 187(1) mT between the $\Delta_{1}$ resonances in Mu-1-HHTT is similar to the splitting observed 
in the corresponding Mu adduct of HAT6 in the Cr phase, which was assumed to be due to differences in the amount of spin density that is transferred to neighboring molecules due to differences in the overlap of the $\pi$-systems of each muoniated radical and the adjacent HAT6 molecules. Our calculations on the HHTT-Mu-1-HHTT-HHTT system indicate that the observed $\Delta_{1}$ splitting in HHTT is much larger than could arise from differences in the amount of spin density transferred to neighboring molecules, which is expected to be $\sim 20 \mathrm{mT}$, so this explanation is incorrect. The maximum $\Delta_{1}$ splitting, based on the calculated dipolar hfccs, that could arise due to different orientations of the Mu-1-HTTT radical with respect to the magnetic field is $94 \mathrm{mT}$, which is significantly less than the observed splitting. A complete and consistent explanation of the ALC- $\mu$ SR spectra of the Cr phase is yet to be available.

At $302 \mathrm{~K}$ there is an additional weak resonance at 1.774(6) T that we have not identified. This resonance disappears above $\sim 310 \mathrm{~K}$. The observed resonance field does not match any of the calculated $\Delta_{0}$ resonance fields for Mu-1-HHTT. We have eliminated the possibility of Mu addition to carbon 2 of the triphenylene ring as our DFT calculations show that $A_{\mu}$ of the resulting radical is $248.01 \mathrm{MHz}$ and $B_{\text {res }}^{\Delta_{1}}=0.911 \mathrm{~T}$.

\section{Resonance linewidth and amplitude in the $\mathrm{Col}_{h}$ and I phases}

The interesting result of the ALC- $\mu \mathrm{SR}$ experiments on HHTT is that there is an extremely large $\Delta_{1}$ resonance in the $\mathrm{Col}_{\mathrm{h}}$ and I phases and that the linewidth and amplitude change significantly with temperature. We will focus on this and demonstrate that this provides information about the interaction of the radicals with neighboring molecules when stacked within a column.

There is a large increase in the width and amplitude of the $\Delta_{1}$ resonance near the $\mathrm{H}-\mathrm{Col}_{\mathrm{h}}$ phase transition, then the width and amplitude of the $\Delta_{1}$ increase with increasing temperature in the $\mathrm{Col}_{\mathrm{h}}$ phase. There is a jump in the $\Delta_{1}$ width at the $\mathrm{Col}_{\mathrm{h}}-\mathrm{I}$ phase transition, while the amplitude begins to decrease. The increase in the width of the $\Delta_{1}$ resonance with increasing temperature in the $\mathrm{Col}_{\mathrm{h}}$ and I phases is very surprising, especially when the lines in the ${ }^{1} \mathrm{H}$ and ${ }^{13} \mathrm{C}$ NMR spectra of HHTT narrow with increasing temperature over the same temperature range due to increased motional averaging $[5,10]$. This unusual behavior is not intrinsic to muoniated radicals as the muoniated spin probes in the calamitic and lyotropic liquid crystals behave like their closed-shell counterparts [14-18,20-23]. Nor is this related to the different timescales probed by $\mu$ SR and NMR. A similar broad $\Delta_{1}$ resonance was observed for the Mu adduct of HAT6 in the $\mathrm{Col}_{\mathrm{h}}$ and I phases [24] and we propose that the same mechanism is at work in both systems.

Many processes can effect the width and amplitude of $\Delta_{1}$ resonances but Kreitzman and Roduner have shown that only electron spin relaxation can lead to broad and intense $\Delta_{1}$ resonances and the suppression of methylene proton $\Delta_{0}$ resonances. Kreitzman and Roduner simulated ALC- $\mu$ SR spectra for a system based on the cyclohexadienyl radical, with $A_{\mu}=526 \mathrm{MHz}$ and $A_{\mathrm{p}}=125 \mathrm{MHz}$, with a range of electron spin relaxation rates $\left(\lambda_{\mathrm{e}}=1 / T_{1}^{\mathrm{e}}\right)$. They found that for moderate electron spin relaxation rates $\left(\lambda_{\mathrm{e}} \leqslant 10 \mu \mathrm{s}^{-1}\right)$ an increase of $\lambda_{\mathrm{e}}$ causes both the width and amplitude to increase, while for

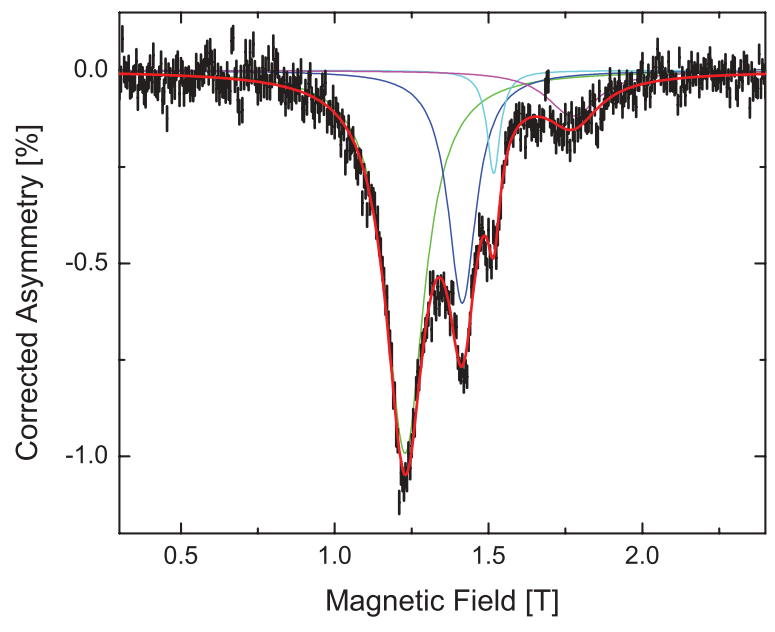

FIG. 8. (Color online) Background-subtracted ALC- $\mu$ SR spectrum of HHTT at $302 \mathrm{~K}$ (Cr phase). The solid lines are the overall fitted spectra.

large $\lambda_{\mathrm{e}}\left(10 \geqslant \lambda_{\mathrm{e}} \geqslant 100 \mu \mathrm{s}^{-1}\right)$ an increase of $\lambda_{\mathrm{e}}$ leads to only broadening of the resonance without a significant decrease in the amplitude This matches our observations in the $\mathrm{H}, \mathrm{Col}_{h}$, and I phases [13]. The increase in the amplitude occurs because $T_{1}^{\mathrm{e}}$ processes bring the radicals formed in the other Zeeman subsystem into resonance. We propose that the increase in the width and amplitude of the $\Delta_{1}$ resonance near the $\mathrm{H}-\mathrm{Col}_{\mathrm{h}}$ phase transition is due to a large increase in $\lambda_{\mathrm{e}}$ and that this increases with increasing temperature in the $\mathrm{Col}_{h}$ and I phases.

In order to verify this we have simulated the effect of electron spin relaxation on the ALC- $\mu$ SR spectrum of Mu1-HHTT using the QUANTUM spin simulation program [34]. We verified that this program was behaving correctly by using the same parameters as Kreitzman and Roduner and obtained identical ALC curves. We have simulated the ALC- $\mu$ SR spectra for unaligned $\mu^{+}-e^{-}-p^{+}$system and ignored the effect molecular wobbling and slow isotropic reorientation, which is known to broaden the $\Delta_{1}$ resonance significantly [18]. The isotropic hfccs used for the simulation were those obtained from the ALC- $\mu$ SR spectra of HHTT and are listed in the caption of Fig. 9. We found that the width of the resonance depends on both $\lambda_{\mathrm{e}}$ and $D_{\mu}^{\|}$and that to get resonances that resemble those observed experimentally we need both parameters to be large. We show the simulations using a muon dipolar hfcc of $-12 \mathrm{MHz}$ as these gave resonances that are in good agreement with the experiments. The resonances are approximately Lorentzian shaped when $\lambda_{\mathrm{e}}$ is much larger than $D_{\mu}^{\|}$. While we cannot determine $\lambda_{\mathrm{e}}$ exactly since we do not know $D_{\mu}^{\|}$, we can conclude from the simulations that $\lambda_{\mathrm{e}}$ is of the order of hundreds of $\mu \mathrm{s}^{-1}$ in the $\mathrm{Col}_{\mathrm{h}}$ and I phases, many times larger than in the $\mathrm{Cr}$ and $\mathrm{H}$ phases.

The increase in the width and decrease in the amplitude of the $\Delta_{1}$ resonance in the I phase compared with the $\mathrm{Col}_{\mathrm{h}}$ phase is most likely caused by slow isotropic reorientation of the radicals on top of the electron spin relaxation. The broadening of $\Delta_{1}$ resonances due to slow isotropic reorientation has been observed for the Mu adduct of cholesterol nonanoate, a chiral nematic LC [18]. The rotational correlation time for the $\mathrm{Mu}$ 1-HHTT radical cannot be determined as the $\Delta_{1}$ line shape 


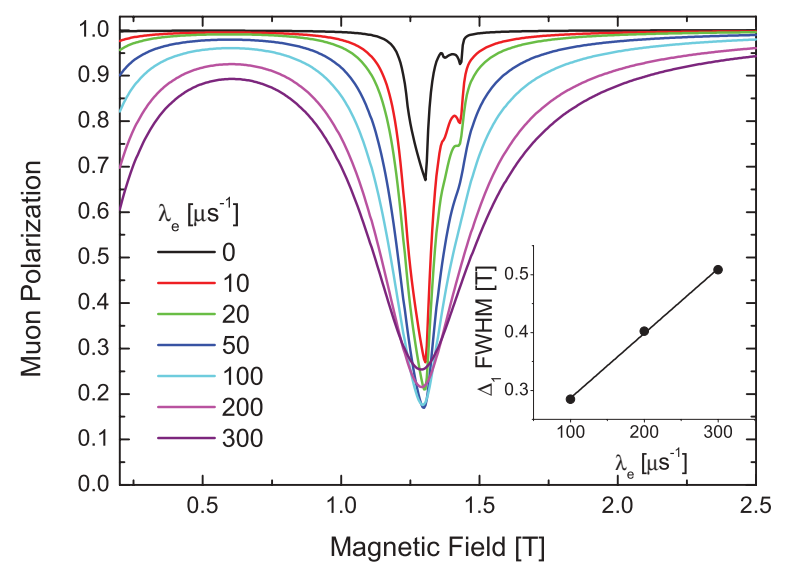

FIG. 9. (Color online) Simulations of the effect of fieldindependent electronic spin relaxation on the avoided level crossing resonance spectrum of $\mathrm{Mu}-1-\mathrm{HHTT}$ radicals that are rapidly rotating about an axis normal to the triphenylene ring and not orientationally ordered. $A_{\mu}^{\text {iso }}=350 \mathrm{MHz}, D_{\mu}^{\|}=-12.0, A_{\mathrm{p}}^{\text {iso }}=86 \mathrm{MHz}$, and $D_{\mathrm{p}}^{\|}=-3.8 \mathrm{MHz}$. The narrowest resonance is the simulation for $\lambda_{\mathrm{e}}=0 \mu \mathrm{s}^{-1}$ and the broadest resonance is the simulation for $\lambda_{\mathrm{e}}=300 \mu \mathrm{s}^{-1}$. The inset graph shows the relationship between the electron spin relaxation rate and the $\Delta_{1}$ FWHM. The simulations were performed using the QUANTUM spin simulation program [34].

depends on both the rotational motion and the electron spin relaxation.

The question that we are left with is what causes the rapid electron spin relaxation. The large increase in the electron spin relaxation rate appears to be related to the formation of columns in which there is significant molecular motion within the column. Columns are assumed to persist in the I phase of HHTT as conductivity measurements of the closely related DLC HAT6 indicate the persistence of molecular stacks some $200 \mathrm{~nm}$ in length in the I phase and neutron scattering measurements of HAT6 also indicated the presence of molecular stacks [35]. The average length of the stacks of HHTT molecules likely changes significantly with temperature, especially around the phase transition, and this could relate to the temperature dependence of $\lambda_{\mathrm{e}}$. The electron spin relaxation is not rapid in the $\mathrm{H}$ phase, even though the radicals are incorporated within columns, because the molecules are essentially frozen on the $\mu \mathrm{SR}$ timescale. We consider several possible causes of electron spin reaction and evaluate whether they could account for the experimental observations.

The possibility of the unpaired electron of Mu-1-HHTT hopping back and forth along the column can be eliminated as $B_{\text {res }}^{\Delta_{1}}$ is not significantly reduced from the value in the $\mathrm{Cr}$ phase, which indicates that there is no reduction in unpaired spin density at the muon as one would expect with long-range diffusion. Moreover it has been shown that the energy of the SOMO in radicals formed by $\mathrm{Mu}$ addition to an aromatic system are considerably different from those of the parent compound and this results in the transfer of an electron between the muoniated radical and a neighboring molecule being very unfavorable [36].

The possibility that the electron spin relaxation is due to the spin-exchange reaction with charge carriers moving up and down the columns is considered remote. This reaction is not important in HAT6 due to the slow diffusion rate of charge carriers $\left(D^{\|} \sim 3.4 \times 10^{-10} \mathrm{~m}^{2} \mathrm{~s}^{-1}\right.$ along the column and $D^{\perp}$ $\sim 5 \times 10^{-13} \mathrm{~m}^{2} \mathrm{~s}^{-1}$ perpendicular to the column axes [37]) and the low concentration of charge carriers as determined by EPR. It is reasonable to suppose that the same is true for HHTT. This reaction could be studied by obtaining ALC- $\mu$ SR spectra of samples with known charge carrier concentrations, produced by exposure of HHTT to iodine vapor as in Ref. [38] or trinitrofluorenone as in Ref. [39].

We propose that the same mechanism is responsible for the rapid electron spin relaxation observed in both HAT6 and HHTT, namely the modulation of the hfecs by the liquidlike motion of the molecules within the columns. This is supported by the DFT calculations described in Sec. III A. The ALC- $\mu$ SR specta of HHTT and HAT6 are similar, except that the $\Delta_{1}$ resonance of HHTT changes significantly with temperature in the $\mathrm{Col}_{\mathrm{h}}$ and I phases while that of HAT6 does not. The difference is most likely due to the molecules having different rotational diffusion rates. This relaxation process should occur for other types of paramagnetic molecules within the columns and the large electron spin relaxation rates should make DLCs unattractive materials for any future spin-based technologies as spin coherence needs to last for at least $10^{4}-10^{6}$ gate operations for the computation to be fault tolerant [40].

\section{CONCLUSION}

$\mathrm{Mu}$ adds to the secondary carbon of HHTT to give a substituted cyclohexadienyl radical. This radical was identified by comparing the positions of the resonances in the ALC- $\mu$ SR spectra with values obtained from DFT calculations. There are multiple overlapping $\Delta_{1}$ and $\Delta_{0}$ resonances in the $\mathrm{Cr}$ phase whose origin has not been conclusively determined. The DFT calculations indicate that differences in the amount of spin density transferred to neighboring molecules, which depends on the relative orientation of the muoniated radical and the HHTT molecules, and different orientations of the radicals with respect to the magnetic field would result in splitting of the $\Delta_{1}$ resonance that is smaller than observed experimentally. There is an extremely broad and intense $\Delta_{1}$ resonance in the $\mathrm{Col}_{\mathrm{h}}$ and I phases that has been interpreted as arising from rapid electron spin relaxation with the rate increasing with increasing temperature. The cause of the electron spin relaxation is most likely the modulation of the hfccs by the liquidlike motion of the molecules within the columns.

\section{ACKNOWLEDGMENTS}

Support from the staff at the Laboratory for Muon Spin Spectroscopy at the Paul Scherrer Institute is gratefully acknowledged. We thank the EPSRC UK for a travel grant to carry out this work. This research project has been supported by the European Commission under the 6th Framework Programme through the Key Action: Strengthening the European Research Area, Research Infrastructures. Contract No. RII3CT-2003-505925. 
[1] P. G. de Gennes and J. Prost, in The Physics of Liquid Crystals, 2nd ed. (Oxford University Press, Oxford, 1993).

[2] P. J. Collings and M. Hird, in Introduction to Liquid Crystals: Chemistry and Physics (Taylor and Francis, Oxford, 1997).

[3] S. Chandrasekhar, in Liquid Crystals, 2nd ed. (Cambridge University Press, Cambridge, 1992).

[4] S. Laschat, A. Baro, N. Steinke, F. Giesselmann, C. Hägele, G. Scalia, R. Judele, E. Kapatsina, S. Sauer, A. Schreivogel et al., Angew. Chem. Int. Ed. 46, 4832 (2007).

[5] S. V. Dvinskikh, J. Thaning, B. Stevensson, K. Jansson, S. Kumar, H. Zimmermann, and A. Maliniak, Phys. Rev. E 74, 021703 (2006).

[6] P. A. Heiney, E. Fontes, W. H. de Jeu, A. Riera, P. Carroll, and A. B. Smith, J. Phys. (France) 50, 461 (1989).

[7] E. Fontes, P. A. Heiney, and W. H. de Jeu, Phys. Rev. Lett. 61, 1202 (1988).

[8] A. M. van de Craats, J. M. Warman, M. P. de Haas, D. Adam, J. Simmerer, D. Haarer, and P. Schuhmacher, Adv. Mater. 8, 823 (1996).

[9] H. Iino, Y. Takayashiki, J.-I. Hanna, R. J. Bushby, and D. Haarer, Appl. Phys. Lett. 87, 192105 (2005).

[10] S. V. Dvinskikh, D. Sandstroem, H. Zimmermann, and A. Maliniak, Prog. Nucl. Magn. Reson. Spectrosc. 48, 85 (2006).

[11] I. McKenzie and E. Roduner, Naturwissenschaften 96, 873 (2009).

[12] E. Meirovitch, Z. Luz, and H. Zimmermann, J. Phys. Chem. 88, 2870 (1984).

[13] S. R. Kreitzman and E. Roduner, Chem. Phys. 192, 189 (1995).

[14] B. W. Lovett, J. Stießberger, S. J. Blundell, T. Jestädt, A. Ardavan, I. M. Marshall, F. L. Pratt, and I. D. Reid, Physica B: Phys. Condensed Matter 289-290, 612 (2000).

[15] B. W. Lovett, S. J. Blundell, J. S. Stießberger, F. L. Pratt, T. Jestädt, W. Hayes, S. P. Cottrell, and I. D. Reid, Phys. Rev. B 63, 054204 (2001).

[16] I. McKenzie, H. Dilger, A. Stoykov, and R. Scheuermann, J. Phys. Chem. B 113, 10135 (2009).

[17] I. McKenzie, H. Dilger, R. Scheuermann, and A. Stoykov, J. Phys.: Conf. Ser. 225, 012034 (2010).

[18] I. McKenzie, R. Scheuermann, K. Sedlak, and A. Stoykov, J. Phys. Chem. B 115, 9360 (2011).

[19] I. McKenzie, R. Scheuermann, A. Stoykov, and K. Sedlak, Phys. Procedia 30, 91 (2012).

[20] R. Scheuermann, I. M. Tucker, A. M. Creeth, H. Dilger, B. Beck, and E. Roduner, Phys. Chem. Chem. Phys. 4, 1510 (2002).
[21] R. Scheuermann, I. M. Tucker, H. Dilger, E. J. Staples, G. Ford, S. B. Fraser, B. Beck, and E. Roduner, Langmuir 20, 2652 (2004).

[22] A. Martyniak, H. Dilger, R. Scheuermann, I. M. Tucker, I. McKenzie, D. Vujosevic, and E. Roduner, Phys. Chem. Chem. Phys. 8, 4723 (2006).

[23] A. Martyniak, H. Dilger, I. McKenzie, R. Scheuermann, J. Lagerwall, and E. Roduner, Colloids Surf. A 309, 224 (2007).

[24] I. McKenzie, A. N. Cammidge, H. Dilger, H. Gopee, R. Scheuermann, A. Stoykov, and U. A. Jayasooriya, Phys. Chem. Chem. Phys. 12, 9900 (2010).

[25] P. W. Percival, B. Addison-Jones, J.-C. Brodovitch, K. Ghandi, and J. Schüth, Can. J. Chem. 77, 326 (1999).

[26] J.-C. Brodovitch, B. Addison-Jones, K. Ghandi, I. McKenzie, P. W. Percival, and J. Schüth, Can. J. Chem. 81, 1 (2003).

[27] J.-C. Brodovitch, K. Ghandi, I. McKenzie, P. W. Percival, and J. Schüth, Physica B (Amsterdam, Neth.) 374-375, 310 (2006).

[28] B. Kohne, W. Poules, and K. Praefcke, Chem. Zeit. 108, 113 (1984).

[29] H. Dilger, A. Martyniak, R. Scheuermann, D. Vujosevic, I. Tucker, I. McKenzie, and E. Roduner, Physica B (Amsterdam, Neth.) 374-375, 317 (2006).

[30] URL http://root.cern.ch.

[31] M. J. Frisch, G. W. Trucks, H. B. Schlegel, G. E. Scuseria, M. A. Robb, J. R. Cheeseman, J. A. Montgomery, Jr., T. Vreven, K. N. Kudin, J. C. Burant et al., in Gaussian 03, Revision C.02 (Gaussian, Wallingford, 2004).

[32] D. Goldfarb, Z. Luz, and H. Zimmermann, J. Chem. Phys. 78, 7065 (1983).

[33] K. Senthilkumar, F. Grozema, F. Bickelhaupt, and L. Siebbeles, J. Chem. Phys. 119, 9809 (2003).

[34] J. S. Lord, Physica B (Amsterdam, Neth.) 374-375, 472 (2006).

[35] F. M. Mulder, J. Stride, S. J. Picken, P. H. J. Kouwer, M. P. de Haas, L. D. A. Siebbeles, and G. J. Kearley, J. Am. Chem. Soc. 125, 3860 (2003).

[36] I. McKenzie, J. Phys. Chem. A 114, 12759 (2010).

[37] N. Boden, R. J. Bushby, and J. Clements, J. Chem. Phys. 98 (1993).

[38] G. B. M. Vaughan, P. A. Heiney, J. P. McCauley, and A. B. Smith, Phys. Rev. B 46, 2787 (1992).

[39] V. S. K. Balagurusamy, S. K. Prasad, S. Chandrasekhar, S. Kuamr, M. Manickam, and C. V. Yelamaggad, Pramana 53, 3 (1999).

[40] I. Žutić, J. Fabian, and S. Das Sarma, Rev. Mod. Phys. 76, 323 (2004). 